\title{
Discriminating invasive adenocarcinoma among lung pure ground-glass nodules: a multi-parameter prediction model
}

\author{
Fuying Hu ${ }^{1,2 \#}$, Haihua Huang ${ }^{3 \#}$, Yunyan Jiang ${ }^{4 \#}$, Minxiang Feng ${ }^{5}$, Hao Wang ${ }^{5}$, Min Tang ${ }^{6}$, Yi Zhou ${ }^{6}$, \\ Xianhua Tan ${ }^{7}$, Yalan Liu ${ }^{8}$, Chen $\mathrm{Xu}^{8}$, Ning Ding ${ }^{2}$, Chunxue Bai ${ }^{2}$, Jie $\mathrm{Hu}^{2}$, Dawei Yang ${ }^{2}$, Yong Zhang ${ }^{2 \wedge}$ \\ ${ }^{1}$ Department of Pulmonary and Critical Care Medicine, The First People's Hospital, Tianmen, China; ${ }^{2}$ Department of Pulmonary and Critical \\ Care Medicine, Zhongshan Hospital, Fudan University, Shanghai, China; ${ }^{3}$ Department of Thoracic Surgery, Shanghai General Hospital, Jiaotong \\ University, Shanghai, China; ${ }^{4}$ Department of Pulmonary and Critical Care Medicine, People's Hospital, Yuxi, China; ${ }^{5}$ Department of Thoracic \\ Surgery, Zhongshan Hospital, Fudan University, Shanghai, China; ${ }^{6}$ Department of Radiology, Zhongshan Hospital, Fudan University, Shanghai, \\ China; ${ }^{7}$ Department of Radiology, The Fifth Hospital of Wuhan, Wuhan, China; ${ }^{8}$ Department of Pathology, Zhongshan Hospital, Fudan University, \\ Shanghai, China \\ Contributions: (I) Conception and design: D Yang, Y Zhang; (II) Administrative support: X Tan, C Bai, J Hu; (III) Provision of study materials or \\ patients: F Hu, Y Jiang, H Huang, M Feng, H Wang, M Tang, Y Zhou, Y Liu, C Xu, N Ding; (IV) Collection and assembly of data: F Hu, Y Jiang, \\ H Huang, D Yang, Y Zhang; (V) Data analysis and interpretation: F Hu, Y Jiang, H Huang, D Yang, Y Zhang; (VI) Manuscript writing: All authors; \\ (VII) Final approval of manuscript: All authors. \\ "These authors contributed equally to this work. \\ Correspondence to: Dawei Yang, MD; Yong Zhang, MD. Department of Pulmonary and Critical Care Medicine, Zhongshan Hospital, Fudan \\ University, Shanghai 200032, China. Email: yang.dawei@zs-hospital.sh.cn; zhang.yong@zs-hospital.sh.cn.
}

Background: Patients with consistent lung pure ground-glass nodules (pGGNs) have a high incidence of lung adenocarcinoma that can be classified as adenocarcinoma in situ (AIS), minimally invasive adenocarcinoma (MIA), or invasive adenocarcinoma (IAC). Regular follow-up is recommended for AIS and MIA, while surgical resection should be considered for IAC. This study sought to develop a multi-parameter prediction model to increase the diagnostic accuracy in discriminating between IAC and AIS or MIA.

Methods: The training data set comprised consecutive patients with lung pGGNs who underwent resection from January to December 2017 at the Zhongshan Hospital. Of the 370 resected pGGNs, 344 were pathologically confirmed to be AIS, MIA, or IAC and were included in the study. The 26 benign pGGNs were excluded. We compared differences in the clinical features (e.g., age and gender), the content of serum tumor biomarkers, the computed tomography (CT) parameters (e.g., nodule size and the maximal CT value), and the morphologic characteristics of nodules (e.g., lobulation, spiculation, pleura indentation, vacuole sign, and normal vessel penetration or abnormal vessel) between the pathological subtypes of AIS, MIA, and IAC. An abnormal vessel was defined as "vessel curve" or "vessel enlargement". Statistical analyses were performed using the chi-square test, analysis of variance (ANOVA), and rank test. The IAC prediction model was constructed via a multivariate logistical regression. Our prediction model for lung pGGNs was further validated in a data set comprising consecutive patients from multiple medical centers in China from July to December 2018. In total, 345 resected pGGNs were pathologically diagnosed as lung adenocarcinoma in the validation data set.

Results: In the training data set, patients with pGGNs $\geq 10 \mathrm{~mm}$ in size had a high incidence $(74.5 \%)$ of IAC. The maximal CT value of IAC $[-416.1 \pm 121.2$ Hounsfield unit $(\mathrm{HU})]$ was much higher than that of MIA $(-507.7 \pm 138.0 \mathrm{HU})$ and AIS $(-602.6 \pm 93.3 \mathrm{HU})(\mathrm{P}<0.001)$. IAC was more common in pGGNs that displayed any of the following CT manifestations: lobulation, spiculation, pleura indentation, vacuole sign, and vessel abnormality. The IAC prediction model was constructed using the parameters that were assessed as risk factors (i.e., the nodule size, maximal CT value, and CT signs). The receiver operating characteristic

^ ORCID: Chunxue Bai, 0000-0001-5798-3130; Yong Zhang, 0000-0003-1631-7048. 
(ROC) analysis showed that the area under the curve (AUC) of this model for diagnosing IAC was 0.910, which was higher than that of the AUC for nodule size alone (0.891) or the AUC for the maximal CT value alone (0.807) $(\mathrm{P}<0.05$, respectively). A multicenter validation data set was used to validate the performance of our prediction model in diagnosing IAC, and our model was found to have an AUC of 0.883 , which was higher than that of the AUC of 0.827 for the module size alone model or the AUC of 0.791 for the maximal CT value alone model $(\mathrm{P}<0.05$, respectively).

Conclusions: Our multi-parameter prediction model was more accurate at diagnosing IAC than models that used only nodule size or the maximal CT value alone. Thus, it is an efficient tool for identifying the IAC of malignant pGGNs and deciding if surgery is needed.

Keywords: Pure ground-glass nodule (pGGN); invasive adenocarcinoma (IAC); prediction model

Submitted May 06, 2021. Accepted for publication Aug 06, 2021.

doi: $10.21037 /$ jtd-21-786

View this article at: https://dx.doi.org/10.21037/jtd-21-786

\section{Introduction}

Lung cancer is the leading malignant tumor threatening human health and accounts for $30 \%$ of total cancer deaths worldwide (1). Fortunately, early stage lung cancer can be detected via low-dose computed tomography (LDCT) screenings in high-risk patients, and the mortality of lung cancer rate is decreasing (2-5). However, the large amounts of lung nodules detected by LDCT have become a new health problem for patients and continue to trouble physicians $(6,7)$. There is an increasing trend of nonsmoking-related lung adenocarcinoma spectrum lesions in the Asian population, most of which are detected as pure ground-glass nodules (pGGNs) in lung cancer LDCT screenings (8-10). On computed tomography (CT) scans, lung pGGNs are the nodules that have low density and a ground-glass opacity with a clear pulmonary vascular texture (11). A high proportion of persistent pGGNs are lung adenocarcinomas (12). PGGNs are recognized as a slowly progressing disease; however, they can display various growth patterns and invasive degrees during the different pathological phases of adenocarcinoma in situ (AIS), minimally invasive adenocarcinoma (MIA), or invasive adenocarcinoma (IAC) (13-16). According to pooled analyses, the 5-year survival rates of AIS and MIA after resection are as high as $100 \%$ and nearly $100 \%$, respectively (17). If resected, IAC with a ground-glass component has a very high 5-year overall survival rate (18). A pGGN $<20 \mathrm{~mm}$ in size is generally AIS or MIA. The National Comprehensive Cancer Network (NCCN) lung cancer screening guidelines of 2020 recommend regular follow-up screenings for patients with these types of pGGN.
Thus, discriminating between IAC and AIS or MIA among lung pGGNs is important in clinical settings. Former studies of the different characteristics of AIS, MIA, and IAC have mainly focused on single CT parameters, such as the nodule size or the CT value $(19,20)$. Recent studies have demonstrated that radiomic features have superior diagnostic performance over conventional features in the diagnosis of IAC in lung sub-solid ground-glass nodules (GGNs) (21). This study sought to develop a multiparameter model using training and validation data sets to improve the diagnostic accuracy of IAC in lung pGGNs and facilitate further clinical-management decisions, such as whether a shorter CT follow-up interval or a biopsy or surgery is required.

We present the following article in accordance with the TRIPOD reporting checklist (available at https://dx.doi. org/10.21037/jtd-21-786).

\section{Methods}

\section{Study design}

From January 2017 to December 2017, a total of 1,579 consecutive patients were clinically diagnosed with malignant lung nodules that were surgically resected at the Zhongshan Hospital, Fudan University, Shanghai, China. Of the 370 pGGNs resected from 309 patients, 344 pGGNs were pathologically confirmed to be lung adenocarcinoma and were enrolled in the training data set (Figure 1A).

The validation data set, which was used after the construction and training of the IAC predicting model was complete, comprised data of patients hospitalized from July 
A

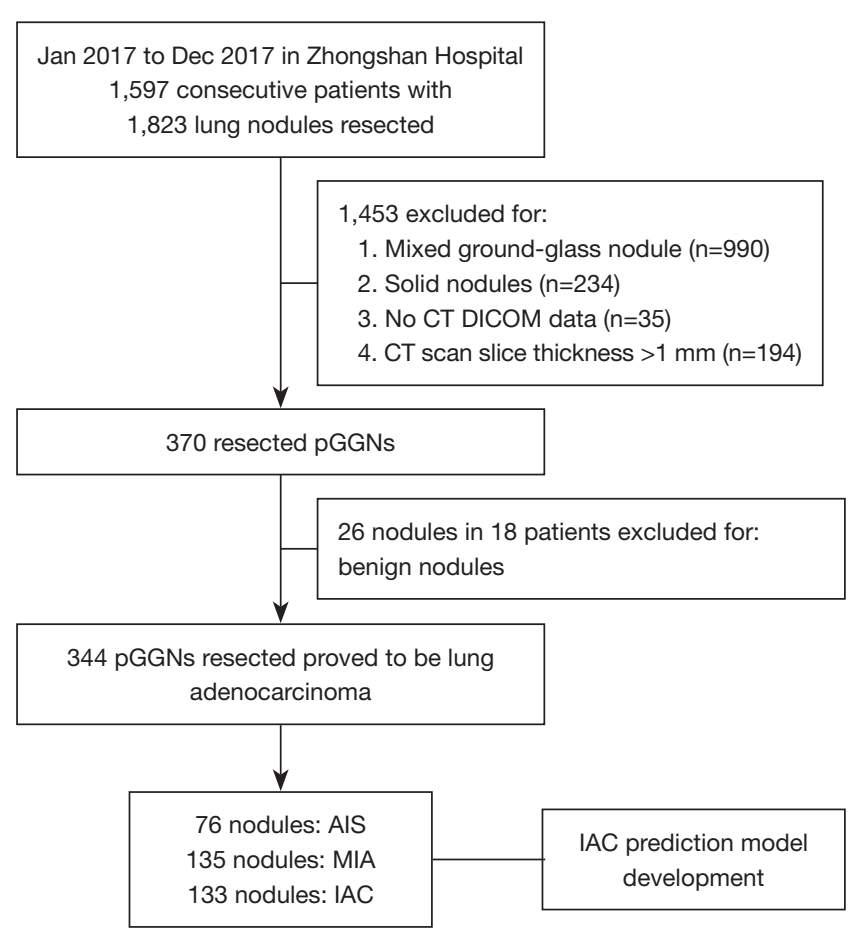

B

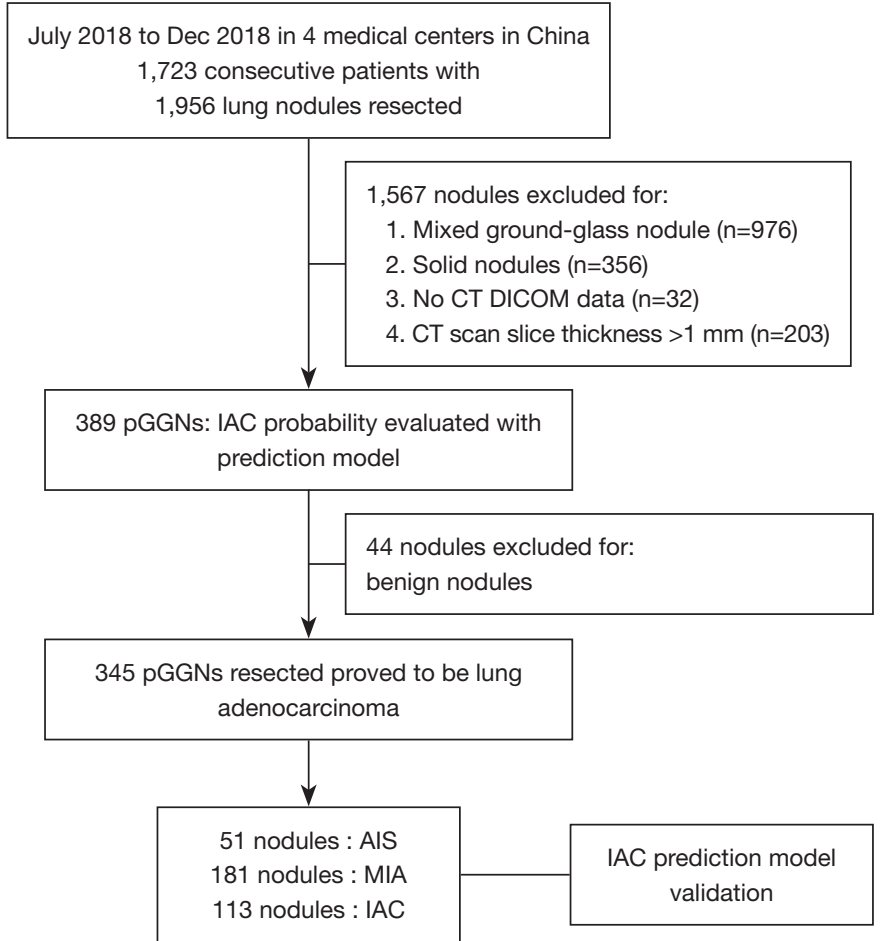

Figure 1 Overview of this study. (A) The training data set comprised 344 malignant pGGNs from Zhongshan Hospital. (B) The validation data set comprised 345 malignant pGGNs from four medical centers in China. CT, computed tomography; pGGNs, pure ground-glass nodules; AIS, adenocarcinoma in situ; MIA, minimally invasive adenocarcinoma; IAC, invasive adenocarcinoma.

to December 2018. A total of 1,723 consecutive patients with clinically diagnosed malignant lung nodules at four medical centers in Shanghai, Wuhan, Yunnan province of China were screened. From these patients, 389 pGGNs were evaluated using the IAC prediction model before resection. In total, 345 resected pGGNs were pathologically diagnosed to be lung adenocarcinoma and were enrolled in the validation data set (Figure 1B). The study was conducted in accordance with the declaration of Helsinki (as revised in 2013). The study was approved by the Institutional Ethics Committee of Zhongshan Hospital (NO. B2017-201), and individual consent for this retrospective analysis was waived.

\section{Patient characteristics}

Clinical information on age and gender was collected. The patients in the training data set had a mean age of 53.4 years (38 patients were aged $\leq 40$ years, 211 were aged $41-60$ years and 95 were aged $>60$ years) (Table 1). Ninety-eight patients were male, and the rest were female. In total, 344 nodules were pathologically assessed. Of these, 76 nodules were confirmed to be AIS, 135 nodules were confirmed to be MIA, and 133 were confirmed to be IAC.

In the validation data set, patients had a mean age of 53.7 years ( 42 patients were aged $\leq 40$ years, 206 were aged $41-60$ years, and 97 were aged $>60$ years) (Table 1 ). Of the 345 pGGNs, 95 were resected from males, and the rest were resected from females. Fifty-one nodules were pathologically confirmed to be AIS, 181 nodules were confirmed to be MIA, and 113 were confirmed to be IAC.

\section{Construction of an IAC prediction model using the training data set}

\section{Serum tumor markers}

The following five serum tumor markers were tested in all participants: carcinoembryonic antigen (CEA), cytokeratin 19 fragment (CYFRA21-1), squamous cell carcinoma antigen (SCC), neuro-specific enolase (NSE), and carbohydrate antigen 19-9 (CA19-9). The serum 
Table 1 The number of pGGNs across the age and gender subgroups (n/total \%) in the training and validation data sets

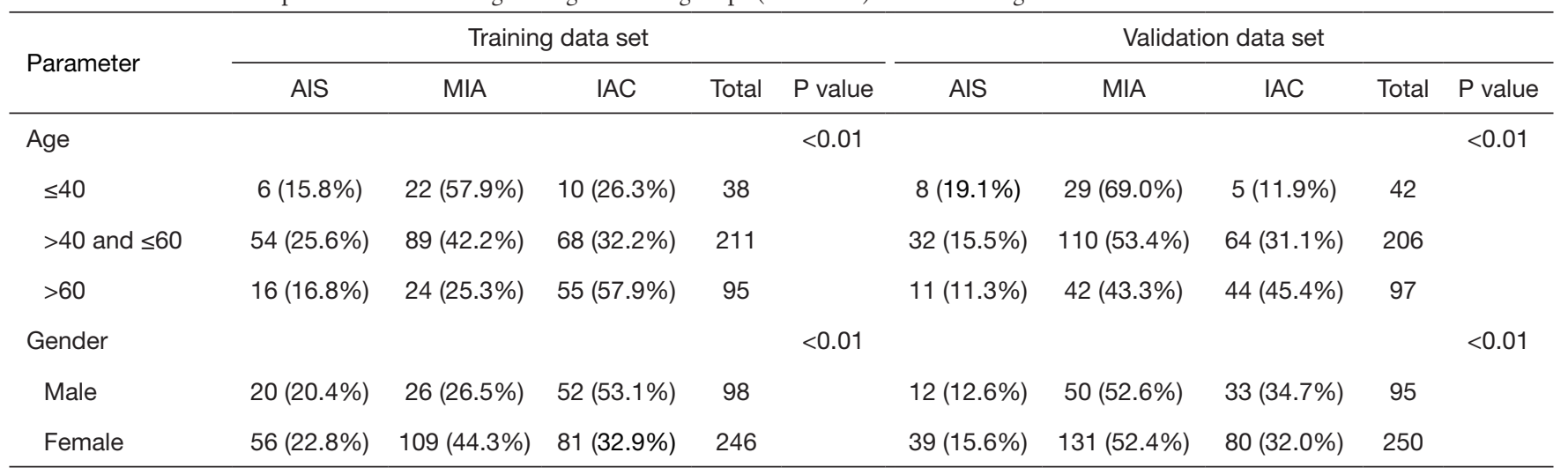

pGGNs, pure ground-glass nodules; AIS, adenocarcinoma in situ; MIA, minimally invasive adenocarcinoma; IAC, invasive adenocarcinoma.

tumor markers were tested with the Elescsys 2010 kits (Roche Corp., Ltd., USA) and analyzed on an Elescsys 2010 electrochemiluminescence immunoanalyzer (Roche Corp., Ltd., USA).

\section{High-resolution computed tomography (HRCT) scans and computer-assisted analyses}

All patients in the training data set underwent a nonenhanced HRCT scan with an image slice thickness of $1 \mathrm{~mm}$ using Siemens Somatom Definition Flash Dual-Source CT. All nodules were pGGNs with a size $\leq 3 \mathrm{~cm}$. All the CT data were recorded as Digital Imaging and Communications in Medicine (DICOM) data, and the images were lung algorithmically rebuilt. The pGGN features assessed included the nodule size (average of the long and short diameters), maximal CT value, and morphological characters of the pGGNs. Vessels were avoided when measuring the CT value. The CT morphological characters of pGGNs included signs of lobulation, spiculation and pleura indentation, vacuole sign, vessel abnormality, and nodule vessel penetration. An abnormal vessel was defined as "vessel curve" or "vessel enlargement". The CT images of the training data set were read, analyzed, and diagnosed with PneuView lung DICOM analysis software 2.3 (Intrasense Corp., Ltd., France) by two attending doctors at the Zhongshan Hospital with more than 15 years of experience in imaging diagnosis who were blind to the pathological results. If any disagreement arose between the doctors, the issue was discussed until a consensus was reached.

\section{Diagnosis of AIS, MIA, and IAC}

The diagnosis of pGGNs in the training data set was determined by the histopathological examination of the resected specimens, and the lesions were classified as AIS, MIA, or IAC according to the 2011 International Association for the Study of Lung Cancer (IASLC), the American Thoracic Society (ATS), the European Respiratory Society (ERS), and the World Health Organization (WHO) classifications of lung neoplasms. Lepidic-predominant adenocarcinomas $\leq 3 \mathrm{~cm}$ in size were classified as AIS, MIA, or IAC. AIS is a non-invasive lesion, MIA has an invasive component $\leq$ of $0.5 \mathrm{~cm}$, and IAC has an invasive component $>0.5 \mathrm{~cm}$. The diagnosis of these nodules was discussed and confirmed by two pathologists at the Zhongshan Hospital with more than 15 years of clinical experience who were blind to the CT predictor factor results.

\section{Statistical analysis}

The data of the training data set were analyzed with SPSS 19.0 (IBM Corp., Armonk, NY, USA). The count data are presented as the number of cases and their percentage. A chi-square test was used to analyze the associations between age, gender, and the content of serum tumor markers with the CT findings, including the signs of lobulation, spiculation and pleural indentation, vacuole sign, vessel abnormalities, and vessel penetration. An analysis of variance (ANOVA) was used to compare the maximal CT values between the various pathological diagnoses. A $\mathrm{P}$ value of $<0.05$ was considered statistically significant. The IAC prediction model was established using nominal dependent variables for stepwise regression with a generalized logit function. A receiver operating characteristic (ROC) analysis was performed to evaluate the performance of the IAC 
prediction model and compare it to models that used only nodule CT value or size.

\section{Evaluation of the IAC prediction model using the validation data set}

The validation data set comprised 345 malignant pGGNs from 4 medical centers in China (i.e., the Zhongshan Hospital, Shanghai, the First People's Hospital, Tianmen City, Hubei Province, the Shanghai General Hospital, Jiaotong University, Shanghai, and the People's Hospital, Yuxi City, Yunnan Province). All patients underwent HRCT scans with an image slice thickness of $1 \mathrm{~mm}$, and the images were lung algorithmically rebuilt. Using the IAC prediction model, we predicted IAC risk of the 389 nodules before surgery. Ultimately, 345 nodules were pathologically confirmed to be lung adenocarcinoma and 44 benign nodules were excluded. The imaging analyses and pathological diagnoses of the pGGNs were performed by local physicians. A ROC analysis was performed to evaluate the performance of the IAC prediction model.

\section{Results}

\section{Construction of the IAC prediction model}

\section{Pathological results for pGGNs}

Thirty-eight-point seven percent of the pathologically confirmed malignant lung pGGNs were IACs. However, most of the IACs of the pGGNs were not highly malignant. Of the 133 IAC cases in the training dataset, there was no nodal and remote metastasis, only 1 case of lymphovascular invasion, and there were 11 cases of pleural invasion (PL1). The pGGNs from older patients or those who were males were more prone to be IACs. The pGGNs from patients aged $>60$ years were more likely to be IACs $(57.9 \%)$ than those from patients aged $\leq 40(26.3 \%)$ or those from patients aged between $40-60$ years $(32.2 \%)(\mathrm{P}<0.01)$. Additionally, while a lower proportion of pGGNs was observed in male than female patients, male patients had a higher incidence of IAC $(53.1 \%)$ than female patients $(32.5 \%$; $\mathrm{P}<0.01)$ (Table 2$)$.

\section{Serum tumor biomarkers}

Our results revealed that the five serum tumor markers had a low capability to diagnose early-stage lung adenocarcinoma from pGGNs. The overall diagnostic sensitivity of CEA, CYFRA21-1, SCC, NSE, and CA19-9 was $1.5 \%, 7.3 \%, 2.6 \%, 11.3 \%$ and $1.2 \%$, respectively.
Additionally, the blood levels of the five tumor biomarkers for AIS, MIA, and IAC did not differ significantly (Table 2).

\section{Associations between CT characteristics and AIS/MIA and IAC}

Larger nodule sizes were strongly associated with an increased risk of IAC. Of the 45 nodules, $<6 \mathrm{~mm}$ in size, the incidence of IAC other than AIS or MIA was as low as $4.4 \%$. Concerning the nodules $6-8,8-10$, and $\geq 10 \mathrm{~mm}$ in size, the incidence of IAC was $7.9 \%, 17.5 \%$, and $74.5 \%$, respectively, indicating an increasing trend. The ROC curve showed that a cut-off value of $9.85 \mathrm{~mm}$ could be used to diagnose IAC with a sensitivity of $85.7 \%$ and a specificity of $81.5 \%$ [area under the curve $(\mathrm{AUC})=0.891]$.

The maximal CT value of the pGGNs was determined without assessing the vascular structure. The maximal CT values of pGGNs diagnosed as AIS, MIA, and IAC were $-602.6 \pm 93.3,-507.7 \pm 138.0$, and $-416.1 \pm 121.2 \mathrm{HU}$, respectively. The variance analysis indicated that the maximal CT value was associated with a risk of IAC $(\mathrm{P}<0.001)$. The ROC curve showed that a maximal CT value of $-482.5 \mathrm{HU}$ diagnosed IAC with a sensitivity of $72.9 \%$ and a specificity of $73.0 \%$ (AUC $=0.807$ ).

Of the pGGNs that displayed lobulation or spiculation, $72.6 \%$ and $63.1 \%$ were identified as IACs, respectively, higher than AIS or MIA $(\mathrm{P}<0.001)$. Pleural indentation $(58.6 \%)$ and vacuole signs (64.0\%) also indicated a higher risk of IAC compared with AIS or MIA $(\mathrm{P}<0.001)$. There was no statistical difference in vessel penetration between IAC and AIS or MIA. However, pGGNs with vessel abnormity had a significantly higher IAC risk $(58.9 \%)$ than those without vessel abnormity $(16.5 \% ; \mathrm{P}<0.01)$ (Table 2$)$.

\section{Methods of IAC prediction model construction}

An IAC prediction model was established using the risk factors defined in the training data set. The results showed that a nodule $\geq 8 \mathrm{~mm}$ in size was a strong predictive factor of IAC (odds ratio: 11.494). Additionally, five signs that could be observed on HRCT, scans (i.e., vessel abnormality, pleural indentation, lobulation, spiculation, and vacuole sign) and the highest CT value were also significant predictive factors of IAC (Figure $2 A, 2 B$ ). Using our logistics regression model, we first calculated the possibility of MIA and IAC compared to AIS, and found that AIS and MIA were similar and difficult to identify. However, the logistics regression model showed a statistically significant difference when IAC was compared to AIS combined with MIA. Thus, a model in which IAC discriminated from 
Table 2 The clinical and CT characteristic differences among AIS, MIA and IAC in the training data set

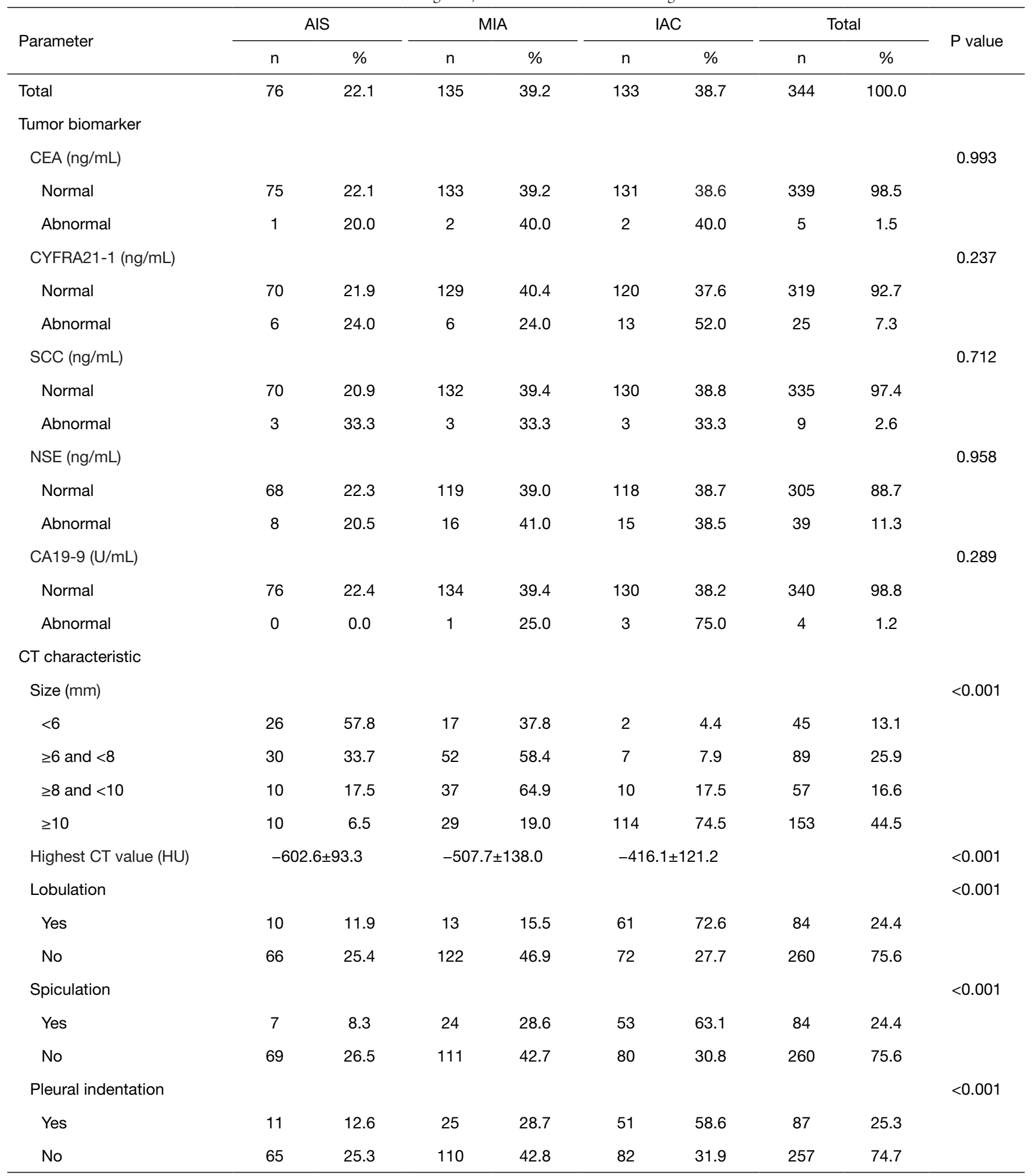

Table 2 (continued) 
Table 2 (continued)

\begin{tabular}{|c|c|c|c|c|c|c|c|c|c|}
\hline Parameter & \multicolumn{2}{|c|}{ AIS } & \multicolumn{2}{|c|}{ MIA } & \multicolumn{2}{|c|}{ IAC } & \multicolumn{2}{|c|}{ Total } & $P$ value \\
\hline Vacuole sign & & & & & & & & & $<0.001$ \\
\hline Yes & 5 & 5.6 & 27 & 30.3 & 57 & 64.0 & 89 & 25.9 & \\
\hline No & 71 & 27.8 & 108 & 42.4 & 76 & 29.8 & 255 & 74.1 & \\
\hline Yes & 56 & 18.0 & 125 & 40.2 & 130 & 41.8 & 311 & 90.4 & \\
\hline No & 20 & 60.6 & 10 & 30.3 & 3 & 9.1 & 33 & 9.6 & \\
\hline Vessel (abnormal) & & & & & & & & & $<0.001$ \\
\hline Yes & 10 & 5.6 & 60 & 33.3 & 106 & 58.9 & 180 & 52.3 & \\
\hline
\end{tabular}

CT, computed tomography; AIS, adenocarcinoma in situ; MIA, minimally invasive adenocarcinoma; IAC, invasive adenocarcinoma; CEA, carcinoembryonic antigen; CYFRA21-1, cytokeratin 19 fragment; SCC, squamous cell carcinoma antigen; NSE, neuro-specific enolase; CA19-9, carbohydrate antigen 19-9; HU, Hounsfield unit.

A

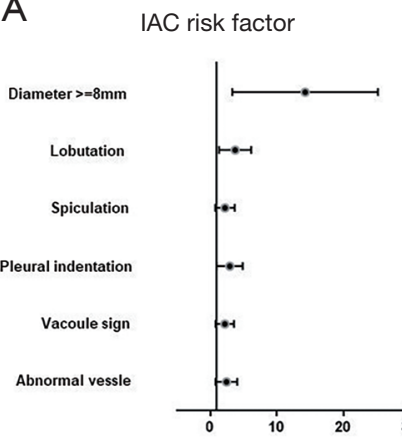

B

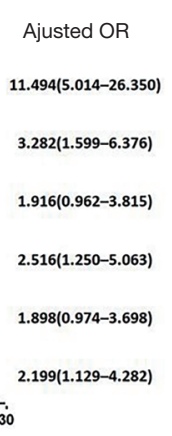

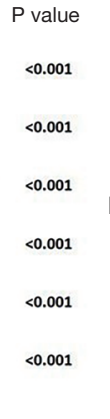

Highest CT value

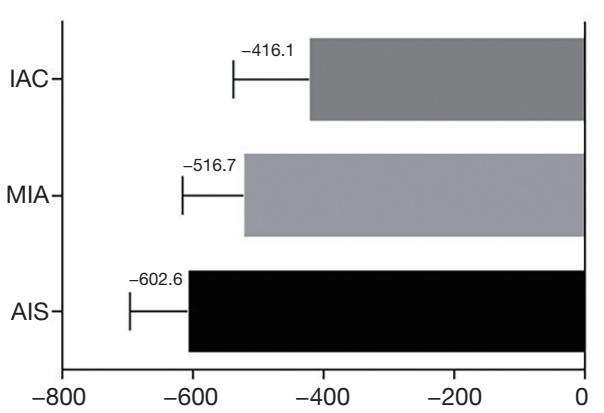

Figure 2 The IAC risk factor for malignant pGGNs in this prediction model. (A) The HRs of six clinical characteristics were used distinguish IAC form AIS or MIA. (B) The different highest CT values of IAC, MIA, and AIS. IAC, invasive adenocarcinoma; CT, computed tomography; pGGNs, pure ground-glass nodules; HRs, hazard ratios; MIA, minimally invasive adenocarcinoma; AIS, adenocarcinoma in situ.

AIS or MIA was successfully established. In the prediction model, a nodule $\geq 8 \mathrm{~mm}$ in size was recorded as "2" and a nodule $<8 \mathrm{~mm}$ in size was recorded as " 1 ". The presence or absence of a specific CT sign in the model was recorded as "1" or "0", respectively. The following function (f) was used to calculate the incidence of IAC (other than AIS or MIA):

$$
\begin{aligned}
f= & 1.9903+1.2209 \times \text { size }(\geq 8 \text { mm or not })+0.00476 \text { CT value }(H U) \\
& +0.5942 \times \text { lobulation }+0.3251 \times \text { spiculation } \\
& +0.3940 \times \text { pleural indentation } \\
& +0.3204 \times \text { vacuole sign }+0.4613 \times \text { abnormal vessel }
\end{aligned}
$$

The possibility of IAC was calculated as $\mathrm{P}$ :

$$
P=\exp (f) /[1+\exp (f)]
$$

Figure 3 shows two typical IAC prediction processes of this model and the pathological results for two lung pGGNs. This model effectively predicted the risk of IAC among pGGNs in the training data set with an AUC as high as 0.910 (CI: 0.879-0.942), which was higher than the AUC of 0.891 for the nodule size alone model or the AUC of 0.807 for the highest $\mathrm{CT}$ value alone model $(\mathrm{P}<0.05$, respectively) (Figure $4 A$ ). The IAC possibility with the cut-off value of 0.42 calculated in the training data set by the IAC prediction model exhibited a high diagnostic efficiency with a sensitivity of $83.7 \%$ and a specificity of $83.9 \%$. The process was easily calculated using the Excel template (Table S1). 

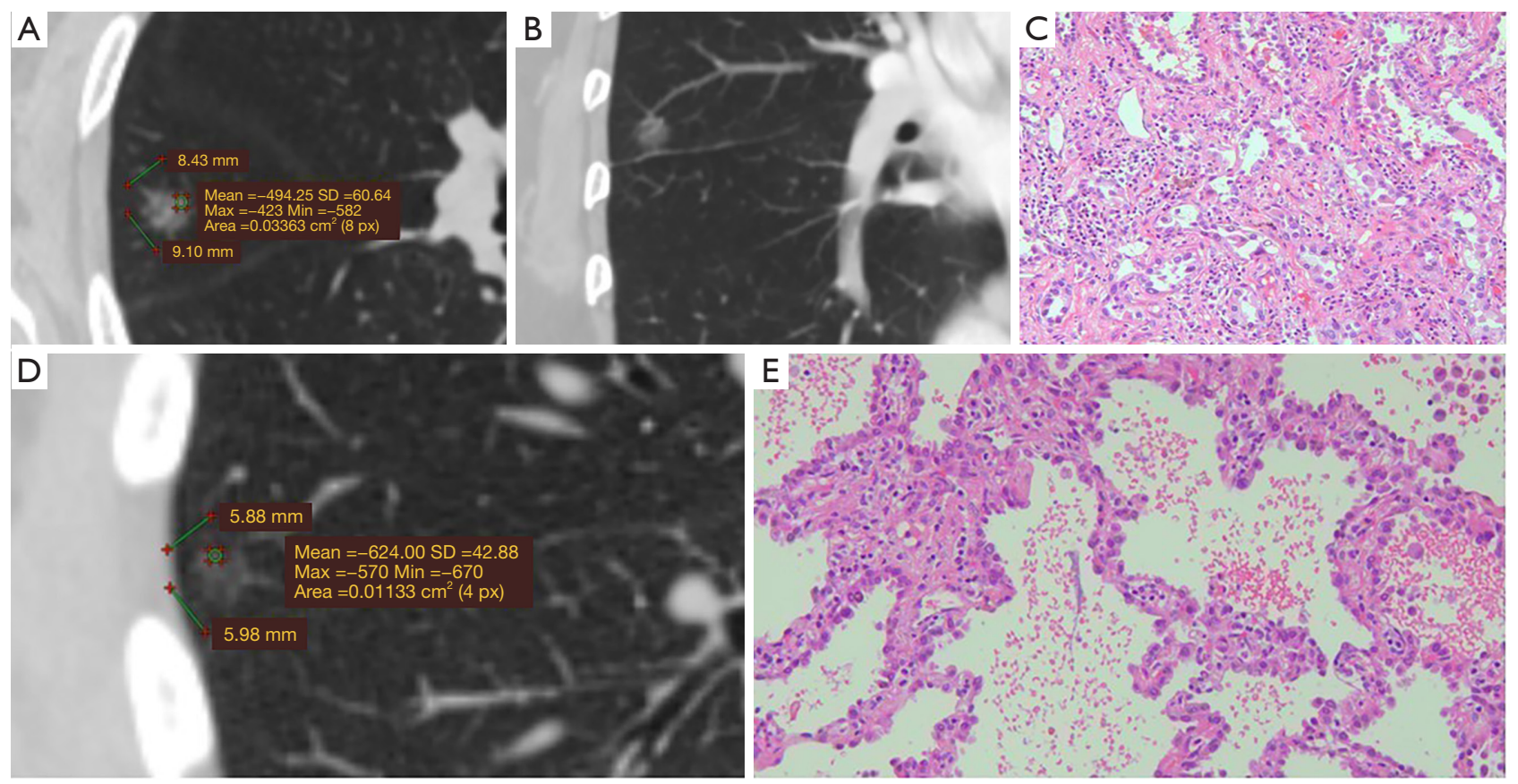

Figure 3 Typical IAC prediction results for two lung pGGNs under this model: one high IAC possibility and one low IAC possibility. (A) PGGN No. 1: $9.10 \times 8.43 \mathrm{~mm}$ in size with a maximum CT value of $-423 \mathrm{HU}$; the predicted IAC possibility was 0.47 (cut off: 0.42 ); (B) typical "vessel curve" of pGGN No.1; (C) pathological result of pGGN No. 1: IAC of acinar type (HE ×200); (D) pGGN No. 2: $5.98 \times 5.88 \mathrm{~mm}$ in size with a normal vessel and a maximum CT value of $-570 \mathrm{HU}$; the predicted IAC possibility was 0.02 (cut off: 0.42 ); (E) pathological result of pGGN No. 2: AIS (HE ×200). IAC, invasive adenocarcinoma; pGGNs, pure ground-glass nodules; CT, computed tomography; HU, Hounsfield unit; AIS, adenocarcinoma in situ.
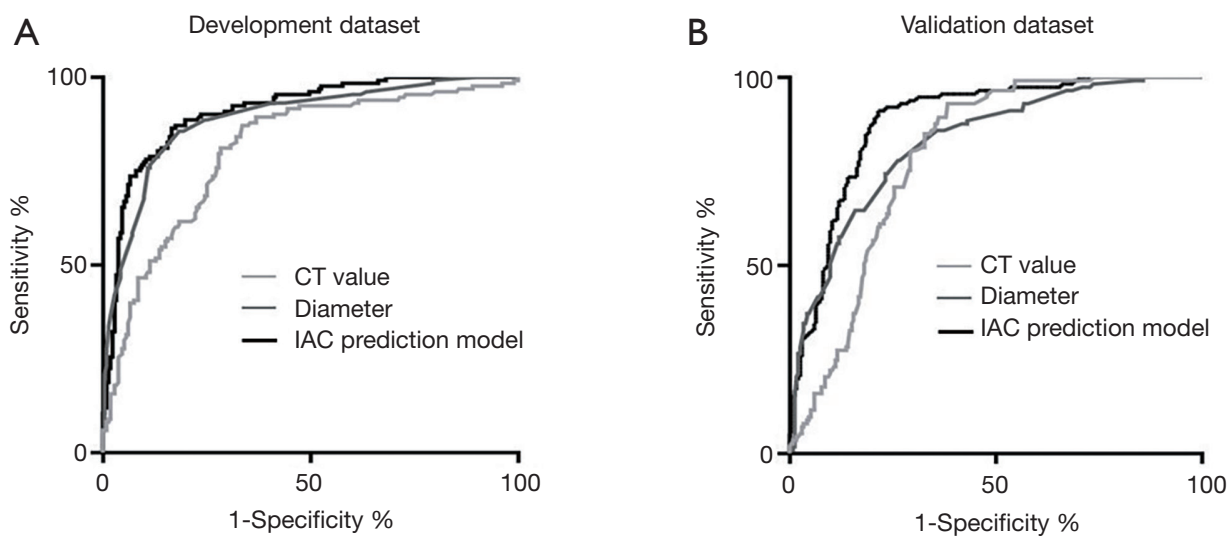

Figure 4 The diagnosis ability of the IAC prediction model in the training and validation data set. (A) The IAC prediction model in the training data set. This model was more accurate at predicting IAC (AUC $=0.910$ ) than models that included size only (AUC $=0.891$ ) or highest CT value only (AUC =0.807). (B) The IAC prediction model in the validation data set. This model was more accurate at predicting IAC (AUC $=0.883$ ) than models that included size only (AUC $=0.827$ ) or highest CT value only (AUC =0.791). CT, computed tomography; IAC, invasive adenocarcinoma; AUC, area under the curve. 


\section{Validation of the IAC prediction model}

The prediction model also performed well in the validation data set and exhibited a significant ability to differentiate between IAC and AIS or MIA. The calculated IAC possibility of the pGGNs was compared to the pathological results; benign nodules were excluded. The AUC of the prediction model was 0.883 (CI: 0.847-0.919), which was higher than that of the nodule size alone model $(\mathrm{AUC}=0.827$ ) or the maximal CT value alone model $(\mathrm{AUC}=0.791)(\mathrm{P}<0.05$, respectively). The IAC possibility with a cut-off value of 0.45 calculated in the validation data set by the IAC prediction model exhibited a high diagnostic efficiency with a sensitivity of $81.4 \%$ and a specificity of $82.3 \%$ (Figure $4 B$ ).

\section{Discussion}

An increasing number of the non-smoker-related early stage lung cancer cases manifest as lung pGGNs in LDCT screenings in Asia. Our view is that the differential diagnosis of lung pGGNs by CT scans is essential to differentiate between IAC and AIS or MIA and thus avoid many unnecessary operations. The present study showed that the percentage of IAC in malignant pGGNs was $38.7 \%$ in the training data set and $32.8 \%$ in the validation data set. Our findings are similar to those of several studies with smaller sample sizes that reported that the possibility of IAC was $16-67 \%$ in malignant lung pGGNs (22-24).

In the training data set, we found that being male and aged $\geq 60$ years were two risk factors of IAC. Notably, these two risk factors have not been well recognized before. These results suggest that clinicians should be alert when pGGNs are found in males or patients aged $\geq 60$ years. Such patients should have CT follow-up scans more frequently, and consideration should be given to whether a biopsy or surgery is necessary.

The present study also showed that the sensitivity of the five serum tumor biomarkers (i.e., CEA, CYFRA21-1, SCC, NSE, and CA19-9) at diagnosing malignant pGGNs was quite low. Indeed, the five serum tumor biomarkers had no significant value in discriminating between AIS, MIA, and IAC. The reason for their ineffectiveness may be that early stage lung adenocarcinoma rarely secrets tumor proteins into the blood.

In the training data set, nodule size was positively correlated with the possibility of IAC. PGGNs $<6 \mathrm{~mm}$ in size were mostly identified as AISs by the pathology results, and only a very few were IACs. IAC was observed in $17.5 \%$ of the pGGNs $8-10 \mathrm{~mm}$ in size and $74.5 \%$ of the pGGNs $\geq 10 \mathrm{~mm}$ in size. Our results are similar to those of another study in China that showed that the possibility of IAC was $88.73 \%$ in pGGNs $\geq 10.5 \mathrm{~mm}$ in size (25). Notably, the incidence of IAC in pGGNs in China is higher than the incidence of IAC in pGGNs of a similar size (39\%) in western countries (24). Previous studies have shown that a GGN size $\geq 10 \mathrm{~mm}$ is the optimal diagnostic threshold for IAC lesion prediction with a high positive prediction value (PPV) (26). Our results also support previous reports that pGGNs $>10 \mathrm{~mm}$ in size showed significantly increased growth than those $<10 \mathrm{~mm}$ in size (27). These results indicate that a biopsy or surgery should primarily be considered when pGGNs are $>10 \mathrm{~mm}$ in size because of the high risk of IAC.

In our study, the maximal CT value was able to predict the invasiveness of lung pGGNs. A previous study confirmed that a mean CT value $<-520 \mathrm{HU}$ was an indicator of alveolar-epithelial atypical hyperplasia (AAH) or AIS rather than MIA (24). Other studies have demonstrated that CT values are significantly different between AAH/AIS and IAC $(19,26)$. The CT value of a pGGN may indicate the invasiveness of the nodule and can serve as a parameter to discriminate between IAC and AIS or MIA.

Previous studies have shown that CT manifestations, such as spiculation and pleural indentation, are important indicators that help clinicians assess the invasiveness of lung nodules before surgery (28-30). Our data showed that the presence of any of the five CT signs (i.e., lobulation, spiculation, pleura indentation, vacuole sign, and vessel abnormality) indicated a higher risk of IAC. Additionally, a combination of these CT signs increased the accuracy of diagnosing IAC in pGGNs.

Finally, using logistic regression and risk factors, we successfully established a multi-parameter IAC prediction model for malignant lung pGGNs. Unlike previous studies, we combined risk factors to increase the CT diagnosis accuracy of IAC in malignant lung pGGNs. Our IAC prediction model based on nodule size, the maximal CT value, and $\mathrm{CT}$ characteristics can more accurately diagnose IAC (AUC $=0.910)$ than models based on nodule size or maximum CT value alone. Further, the diagnostic ability of our model was validated using a multicenter validation data set for which our model also exhibited higher accuracy (AUC $=0.883$ ) than models that were based on nodule size or maximum CT value alone. Our multi-parameter IAC prediction model can improve the accuracy of diagnosing IAC from lung pGGNs, inform clinical decisions about 
whether further management is needed, and ensure that unnecessary operations for pGGNs of AIS or MIA are avoided.

This study had several limitations. First, due to a selection bias, the patients (nodules) were more likely to be at high risk of IAC and undergo surgery. Second, the definition of pGGNs and the criteria for pathological diagnosis may differ among physicians. Third, the subjectivity of radiologists may result in different judgments of the CT characteristics, such as vessel curve or vessel enlargement, of lung nodules. Finally, it is possible that there was a deviation in the $\mathrm{CT}$ value measurement as different software or CT rebuilding methods were used. These limitations may reduce the diagnosis accuracy of IAC in this prediction model and should be considered in clinical applications.

\section{Conclusions}

The present study showed that clinical information and CT characteristics were predictors of IAC in patients with malignant lung pGGNs. This multi-parameter prediction model appears to be more accurate at identifying IAC in malignant lung pGGNs than the formerly recommended single-parameter models that are based on nodule size or CT value alone. This model can inform clinical decisions as to whether a biopsy or surgery is needed for lung pGGNs.

\section{Acknowledgments}

We would like to thank TopEdit and AME editing service for linguistic assistance in preparing this manuscript. We would also like to thank Dr. Xianhua Tan for his definition of a "vessel curve" for lung nodule diagnosis in CT scans.

Funding: This work was supported by The National Nature Science Foundation of China (31400713, 82170110), the Shanghai Top-Priority Clinical Key Discipline Construction Project (2017ZZ02013), the Shanghai Pujiang Program (20PJ1402400) and the Shanghai Engineer \& Technology Research Center of Internet of Things for Respiratory Medicine (20DZ2254400).

\section{Footnote}

Reporting Checklist: The authors have completed the STROBE reporting checklist. Available at https://dx.doi. org/10.21037/jtd-21-786
Data Sharing Statement: Available at https://dx.doi. org/10.21037/jtd-21-786

Peer Review File: Available at https://dx.doi.org/10.21037/ jtd-21-786

Conflicts of Interest: All authors have completed the ICMJE uniform disclosure form (available at https://dx.doi. org/10.21037/jtd-21-786). The authors have no conflicts of interest to declare.

Ethical Statement: The authors are accountable for all aspects of the work, including ensuring that any questions related to the accuracy or integrity of any part of the work have been appropriately investigated and resolved. The study was conducted in accordance with the declaration of Helsinki (as revised in 2013). The study was approved by the Institutional Ethics Committee of Zhongshan Hospital (NO. B2017-201), and individual consent for this retrospective analysis was waived.

Open Access Statement: This is an Open Access article distributed in accordance with the Creative Commons Attribution-NonCommercial-NoDerivs 4.0 International License (CC BY-NC-ND 4.0), which permits the noncommercial replication and distribution of the article with the strict proviso that no changes or edits are made and the original work is properly cited (including links to both the formal publication through the relevant DOI and the license). See: https://creativecommons.org/licenses/by-nc-nd/4.0/.

\section{References}

1. Siegel RL, Miller KD, Jemal A. Cancer statistics, 2020. CA Cancer J Clin 2020;70:7-30.

2. Wiener RS, Gould MK, Arenberg DA, et al. An official American Thoracic Society/American College of Chest Physicians policy statement: implementation of lowdose computed tomography lung cancer screening programs in clinical practice. Am J Respir Crit Care Med 2015;192:881-91.

3. Sadate A, Occean BV, Beregi JP, et al. Systematic review and meta-analysis on the impact of lung cancer screening by low-dose computed tomography. Eur J Cancer 2020;134:107-14.

4. National Lung Screening Trial Research Team; Aberle DR, Adams AM, et al. Reduced lung-cancer mortality with low-dose computed tomographic screening. N Engl J Med 
2011;365:395-409.

5. Huang KL, Wang SY, Lu WC, et al. Effects of lowdose computed tomography on lung cancer screening: a systematic review, meta-analysis, and trial sequential analysis. BMC Pulm Med 2019;19:126.

6. Heleno B, Siersma V, Brodersen J. Estimation of Overdiagnosis of Lung Cancer in Low-Dose Computed Tomography Screening: A Secondary Analysis of the Danish Lung Cancer Screening Trial. JAMA Intern Med 2018;178:1420-2.

7. Patz EF Jr, Pinsky P, Gatsonis C, et al. Overdiagnosis in low-dose computed tomography screening for lung cancer. JAMA Intern Med 2014;174:269-74.

8. Wu FZ, Huang YL, Wu CC, et al. Assessment of Selection Criteria for Low-Dose Lung Screening CT Among Asian Ethnic Groups in Taiwan: From Mass Screening to Specific Risk-Based Screening for Non-Smoker Lung Cancer. Clin Lung Cancer 2016;17:e45-56.

9. Lin KF, Wu HF, Huang WC, et al. Propensity score analysis of lung cancer risk in a population with high prevalence of non-smoking related lung cancer. BMC Pulm Med 2017;17:120.

10. Wu FZ, Huang YL, Wu YJ, et al. Prognostic effect of implementation of the mass low-dose computed tomography lung cancer screening program: a hospitalbased cohort study. Eur J Cancer Prev 2020;29:445-51.

11. Okada T, Iwano S, Ishigaki T, et al. Computer-aided diagnosis of lung cancer: definition and detection of ground-glass opacity type of nodules by high-resolution computed tomography. Jpn J Radiol 2009;27:91-9.

12. Kim HS, Lee HJ, Jeon JH, et al. Natural history of ground-glass nodules detected on the chest computed tomography scan after major lung resection. Ann Thorac Surg 2013;96:1952-7.

13. Takahashi S, Tanaka N, Okimoto T, et al. Long term follow-up for small pure ground-glass nodules: implications of determining an optimum follow-up period and high-resolution CT findings to predict the growth of nodules. Jpn J Radiol 2012;30:206-17.

14. Lee HW, Jin KN, Lee JK, et al. Long-Term Follow-Up of Ground-Glass Nodules After 5 Years of Stability. J Thorac Oncol 2019;14:1370-7.

15. Cho J, Kim ES, Kim SJ, et al. Long-Term Follow-up of Small Pulmonary Ground-Glass Nodules Stable for 3 Years: Implications of the Proper Follow-up Period and Risk Factors for Subsequent Growth. J Thorac Oncol 2016;11:1453-9.

16. Tang EK, Chen CS, Wu CC, et al. Natural History of
Persistent Pulmonary Subsolid Nodules: Long-Term Observation of Different Interval Growth. Heart Lung Circ 2019;28:1747-54.

17. Behera M, Owonikoko TK, Gal AA, et al. Lung Adenocarcinoma Staging Using the 2011 IASLC/ATS/ ERS Classification: A Pooled Analysis of Adenocarcinoma In Situ and Minimally Invasive Adenocarcinoma. Clin Lung Cancer 2016;17:e57-64.

18. Suzuki K, Watanabe SI, Wakabayashi M, et al. A singlearm study of sublobar resection for ground-glass opacity dominant peripheral lung cancer. J Thorac Cardiovasc Surg 2020. [Epub ahead of print]. doi: 10.1016/ j.jtcvs.2020.09.146.

19. Ichinose J, Kawaguchi Y, Nakao M, et al. Utility of Maximum CT Value in Predicting the Invasiveness of Pure Ground-Glass Nodules. Clin Lung Cancer 2020;21:281-7.

20. Lee GD, Park CH, Park HS, et al. Lung Adenocarcinoma Invasiveness Risk in Pure Ground-Glass Opacity Lung Nodules Smaller than $2 \mathrm{~cm}$. Thorac Cardiovasc Surg 2019;67:321-8.

21. Wu YJ, Liu YC, Liao CY, et al. A comparative study to evaluate CT-based semantic and radiomic features in preoperative diagnosis of invasive pulmonary adenocarcinomas manifesting as subsolid nodules. Sci Rep 2021;11:66.

22. Heidinger BH, Anderson KR, Nemec U, et al. Lung Adenocarcinoma Manifesting as Pure Ground-Glass Nodules: Correlating CT Size, Volume, Density, and Roundness with Histopathologic Invasion and Size. J Thorac Oncol 2017;12:1288-98.

23. Son JY, Lee HY, Kim JH, et al. Quantitative CT analysis of pulmonary ground-glass opacity nodules for distinguishing invasive adenocarcinoma from non-invasive or minimally invasive adenocarcinoma: the added value of using iodine mapping. Eur Radiol 2016;26:43-54.

24. Lim HJ, Ahn S, Lee KS, et al. Persistent pure ground-glass opacity lung nodules $\geq 10 \mathrm{~mm}$ in diameter at CT scan: histopathologic comparisons and prognostic implications. Chest 2013;144:1291-9.

25. Jin X, Zhao SH, Gao J, et al. CT characteristics and pathological implications of early stage (T1N0M0) lung adenocarcinoma with pure ground-glass opacity. Eur Radiol 2015;25:2532-40.

26. Wu FZ, Chen PA, Wu CC, et al. Semiquantative Visual Assessment of Sub-solid Pulmonary Nodules $\leq 3 \mathrm{~cm}$ in Differentiation of Lung Adenocarcinoma Spectrum. Sci Rep 2017;7:15790.

27. Lee JH, Park CM, Lee SM, et al. Persistent pulmonary 
subsolid nodules with solid portions of $5 \mathrm{~mm}$ or smaller: Their natural course and predictors of interval growth. Eur Radiol 2016;26:1529-37.

28. Liu LH, Liu M, Wei R, et al. CT findings of persistent pure ground glass opacity: can we predict the invasiveness? Asian Pac J Cancer Prev 2015;16:1925-8.

29. Nambu A, Araki T, Taguchi Y, et al. Focal area of groundglass opacity and ground-glass opacity predominance on

Cite this article as: $\mathrm{Hu} F$, Huang $\mathrm{H}$, Jiang $\mathrm{Y}$, Feng $M$, Wang H, Tang M, Zhou Y, Tan X, Liu Y, Xu C, Ding N, Bai C, Hu J, Yang D, Zhang Y. Discriminating invasive adenocarcinoma among lung pure ground-glass nodules: a multi-parameter prediction model. J Thorac Dis 2021;13(9):5383-5394. doi: $10.21037 /$ jtd-21-786 thin-section CT: discrimination between neoplastic and non-neoplastic lesions. Clin Radiol 2005;60:1006-17.

30. Yanagawa M, Johkoh T, Noguchi M, et al. Radiological prediction of tumor invasiveness of lung adenocarcinoma on thin-section CT. Medicine (Baltimore) 2017;96:e6331.

(English Language Editors: L. Huleatt and J. Chapnick) 
Supplementary

Table S1 The excel template for calculating IAC risk of lung malignant pGGN

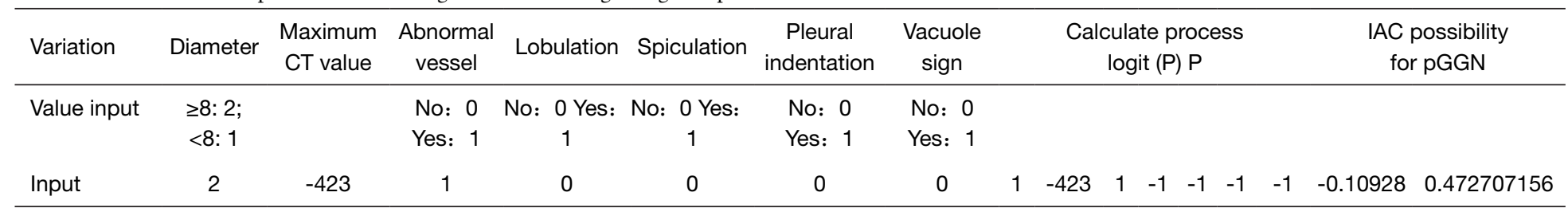

$\mathrm{CT}$, computed tomography; IAC, invasive adenocarcinoma; pGGN, pure ground-glass nodules. 